\title{
Experimental study of low-frequency oscillations and large-scale circulations in turbulent mixed convection
}

\author{
Andreas Westhoff*, Johannes Bosbach, Daniel Schmeling, Claus Wagner \\ German Aerospace Center (DLR) Göttingen, Institute of Aerodynamics and Flow Technology, Bunsenstr. 10, D-37073 Göttingen, Germany
}

\section{A R T I C L E I N F O}

\section{Article history:}

Received 7 December 2009

Received in revised form 21 April 2010

Accepted 28 April 2010

Available online $\mathrm{xxxx}$

\section{Keywords:}

Large-scale circulations

Particle image velocimetry

Proper orthogonal decomposition

Turbulent mixed convection

Forced convection

Low-frequency oscillations

\begin{abstract}
A B S T R A C T
The formation and dynamics of large-scale circulations in forced and mixed convection has been studied at ambient and elevated fluid pressure by means of particle image velocimetry and temperature measurements. The study has been conducted in two rectangular containers of the same shape and aspect ratios of $\Gamma_{x z}=1$ and $\Gamma_{y z}=5$. For the measurements at high fluid pressure the dimensions of the cell have been scaled down by a factor of 5 . Air with $P r=0.7$ has been used as fluid in both configurations. Forced convection has been investigated at $R e=1.01 \times 10^{4}$ and mixed convection has been studied at $A r=3.3$, $R e=1.01 \times 10^{4}$ and $R a=2.4 \times 10^{8}$. In this configuration low-frequency oscillations in the heat transfer between the inlet and outlet have been found for mixed convection. Instantaneous velocity vector fields obtained from particle image velocimetry have been analysed using proper orthogonal decomposition and an algorithm to detect the core and the core centre position of large-scale circulations.
\end{abstract}

(c) 2010 Elsevier Inc. All rights reserved.

\section{Introduction}

In many large-scale convective flows the transport of heat strongly depends on the dynamics of large-scale flow structures. In mixed convection (MC) the heat transfer is further determined by the interaction of forced convection (FC) and thermal convection (TC). Particularly turbulent MC has gained to a lot of interest during the last decades and is of utmost importance, e.g. in geophysics, astrophysics (Kupka, 2003), indoor climatisation (Costa et al., 2000; Linden, 1999) or in industrial processes and applications (Sillekens et al., 1998).

The motivation of the current study is to improve the understanding of the physical mechanisms which drive the flow structure formation and heat transport in turbulent MC. Our main attention is given to the inquiry of:

- How does the flow structure formations depend on the dimensionless parameters, particularly the ratio of buoyancy and inertia forces?

- How do structure and dynamics of the large-scale circulation (LSC) influence the global heat transfer?

- How far can the spatial dimensions of MC be scaled by using classical concepts, e.g. by adjustment of fluid pressure and inflow velocity (Westhoff et al., 2007, 2008)?

\footnotetext{
* Corresponding author.

E-mail address: andreas.westhoff@dlr.de (A. Westhoff).
}

The present study addresses mainly the second issue; the formation and dynamics of the LSC and their influence on the heat transfer is studied experimentally in a rectangular container, i.e. a fluid layer, which is heated from below, cooled from above, and further exposed to forced convection under well-defined conditions.

This system is characterised by five dimensionless parameters, i.e. the Rayleigh number $R a \equiv \Delta T \beta g H^{3} \kappa / v$, the Reynolds number $R e \equiv U H / v$, the Prandtl number $\operatorname{Pr} \equiv v / \kappa$ and the aspect ratios of the rectangular container $\Gamma_{x z} \equiv W / H$ and $\Gamma_{y z} \equiv L / H$. Here $\beta$ denotes the isobaric thermal expansion coefficient, $g$ the acceleration due to gravity, $\Delta T$ the applied temperature difference, $\kappa$ the thermal diffusivity, $v$ the kinematic viscosity, $U$ the characteristic velocity, $W$ the width, $L$ the length, and $H$ the height of the cell. An additional parameter to describe mixed convection is the Archimedes number $A r=R a /\left(R e^{2} \times P r\right)=\Delta T \beta g H / U^{2}$, which is the ratio of buoyancy and inertia forces. For $A r \ll 1$ the flow is primarily driven by inertia forces, while for $A r \gg 1$ the flow is dominated by buoyancy forces. Flows with $A r \approx 1$ are termed MC.

The limiting case of pure TC is still under heavy investigation. Most of the studies consider the so-called Rayleigh-Bénard convection (RBC), where a fluid layer is confined between two horizontal parallel plates heated from below and cooled from above. In $\mathrm{RBC}$ the transport of heat often leads to the generation of thermal "plumes", which are emitted as hot plumes from the bottom thermal boundary layer and as cold plumes from the top thermal boundary layer. In a broad range of $R a$ these plumes drive LSC structures. The understanding of the motion of the plumes and 
the resulting LSCs remains one of the challenging problems in research of convective flows. Qui and Tong (2001a), e.g. provided a systematic parameter study of the formation of LSCs in a cylindrical cell with varying aspect ratios for $4.9 \times 10^{8} \leqslant R a \leqslant 3.28 \times 10^{10}$ with water as working fluid by means of laser doppler velocimetry. They also studied the onset of coherent oscillations in turbulent RBC (Qui and Tong, 2001b) in a cylindric container with an aspect ratio of one. Another study focusing on the plume motion and LSC in a cylindrical cell has been performed by Funfschilling and Ahlers (2004). They found in horizontal direction oscillations of the plume motion and a mean wind oscillating periodically in time with a unique frequency over hundreds of cycles. A study more closely related to our study has been presented by Xia et al. (2003). They investigated the formation of spatial structures in RBC of water for $R a=9.0 \times 10^{8}-9.0 \times 10^{9}$ in a rectangular geometry by means of Particle Image Velocimetry (PIV). Another study of LSCs formation has been presented by Niemela et al. (2001). They experimentally explored the dynamics of the wind in convection of helium in a cylindric apparatus of aspect ratio unity up to high $R a$ of about $10^{16}$.

In systems with a high degree of symmetry such LSCs tend to exhibit low frequency dynamics, such as e.g. rotations, cessations or torsional oscillations (Funfschilling et al., 2008). In MC low-frequency oscillations of the heat transfer between air inlet and outlet have been observed and found to be the result of the dynamics of coherent LSCs (Westhoff et al., 2008). In the paper presented here MC is studied for $A r=3.3$ by means of PIV and local temperature measurements. In order to identify the characteristic frequencies of the LSC dynamics the two-dimensional three-component (2D3C) PIV results are analysed using Proper Orthogonal Decomposition (POD).

\section{Experimental set-up}

In the following section, the experimental apparatus and the measurement methods are described. Further the methods used to analyse of the coherent structures are introduced.

\subsection{The enclosures}

To cover a large parameter range of $600<R e<3 \times 10^{6}$ and $1 \times 10^{5}<R a<1 \times 10^{11}$ two convection cells with a squared cross section and an aspect ratio of $\Gamma_{x y}=1$ and $\Gamma_{x z}=5$ have been constructed using air as working fluid under different pressure conditions (Fig. 1). The cells are equipped with an air inlet at the top and an air outlet at the bottom. The in- and outlet channels have a rectangular cross section, are located at the same side of the cell and span the whole length of the cell. The inlet channel has a height of $H_{\text {in }}=\frac{1}{20} \times H$ and a length of $L_{\text {in }}=30 \times H_{\text {in }}$ to assure a well-defined and a fully developed channel flow, while the outlet channel has a height of $H_{\text {out }}=\frac{3}{5} \times H_{\text {in }}$ and a length of $L_{\text {out }}=30 \times H_{\text {out }}$. All side walls are thermally insulated by a layer system with an insulating sheath of air between two transparent windows. Hence, we nearly realise adiabatic boundary conditions while maintaining the optical accessibility of the cell. The bottom is equipped with a heating plate and the top with a heat exchanger consisting of an aluminium body with cooling fins. One of the cells with the dimensions $W \times H \times L=0.1 \mathrm{~m} \times 0.1 \mathrm{~m} \times 0.5 \mathrm{~m}$ was designed to be operated under high pressure conditions of up to 100 bar. The measurements under high pressure conditions were performed in the High Pressure Wind Tunnel Göttingen (HDG), which is a Göttingen-type closed circuit low speed wind tunnel that can be pressurised up to 100 bar. For the experiments the function of the HDG is threefold. First it allows to adjust the fluid pressure, second it provides the inflow to the convection cell, and finally it supplies cooling to the cell ceiling. The second convection cell has been constructed to work under ambient pressure conditions with the same aspect ratio, but its dimensions are scaled up by a factor of five. For more details of the experimental set-ups the reader is referred to Schmeling et al. (2010) and Westhoff et al. (2007).

\subsection{Particle image velocimetry and temperature measurement}

In this article PIV results obtained in the experiments under ambient pressure are presented. Two-dimensional two-component (2D2C) PIV has been carried out in different cross sections of the cell. Additionally two-dimensional three-component (2D3C) PIV has been performed in a longitudinal cross section. The conducted 2D2C measurement planes are located at $X=0.5 \times L, X=0.625 \times L$, $X=0.75 \times L$ and $X=0.9 \times L$ and the $2 \mathrm{D} 3 C$ cross section is located at $Z=0.5 \times H$. The instantaneous velocity fields have been acquired with a repetition rate of $2 / 3 \mathrm{~Hz}$. More details on the PIV set-up can be found in Schmeling et al. (2010).

For the FC case, which has been measured for comparison, 800 instantaneous velocity fields have been evaluated for every measurement plane. Correspondingly, for MC 4800 instantaneous velocity fields have been measured. Each instantaneous velocity field comprises between 30,000 and 70,000 velocity vectors.

The reason for the much lower number of instantaneous velocity fields measured at FC in contrast to the MC case is that the flow in the FC case is much less instationary and thus 800 velocity fields are sufficient to obtain converged and reliable flow statistics. This can be clearly identified by looking at the spatially averaged difference between the time averaged velocity fields up to the $n$th image $\mathfrak{u}^{(n)}$ and the average field $\mathfrak{U}$ of all $N$ velocity fields

$\Delta \mathfrak{u}^{(\mathrm{n})}=\frac{1}{M} \sum_{\mathrm{m}=1}^{M}\left|\mathfrak{u}_{\mathrm{m}}^{(\mathrm{n})}-\mathfrak{U}_{\mathrm{m}}\right|$.

$M$ denotes the number of vectors in each of the $N$ velocity fields. Further, $\Delta \mathfrak{u}^{(\mathrm{n})}$ is normalised by the spatially averaged velocity magnitude $\widehat{\mathfrak{U}}=1 / M \sum_{M} \mathfrak{U}_{\mathrm{m}}$. For FC (Fig. 2a) and MC (Fig. 2b) $\Delta \mathfrak{u} / \widehat{\mathfrak{U}}$ is already less than $1.5 \%$ for $n \approx 1 / 4 \times N$ velocity fields. Moreover, after averaging of more than $n=1 / 4 \times N$ velocity fields $\Delta \mathfrak{u}$ is nearly monotonically nonincreasing. Additionally, the convergence considering a test statistic with a standard deviation of $\tilde{\sigma}^{(\mathrm{n})}=\sigma_{\mathfrak{l l}}^{(\mathrm{n})} \sqrt{n}$ has been shown. Here $\sigma_{2 \mathfrak{r}}$ denotes the standard deviation of the velocity magnitude. The convergence behaviour of $\widetilde{\sigma}^{(n)}$, normalised with the spatially averaged velocity magnitude $\widehat{\mathfrak{u}}^{(\mathrm{n})}$, is shown in the insets of
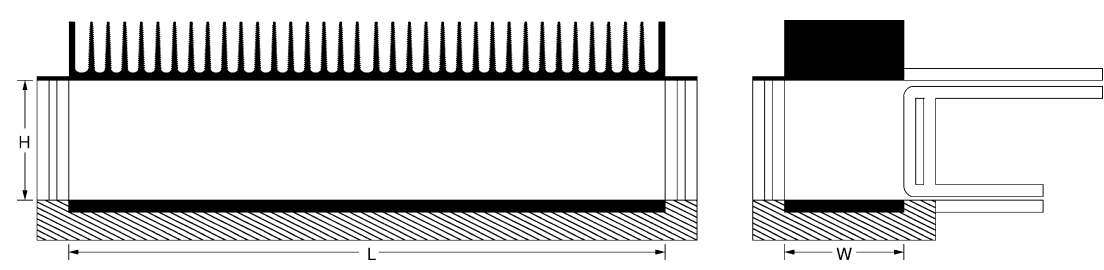

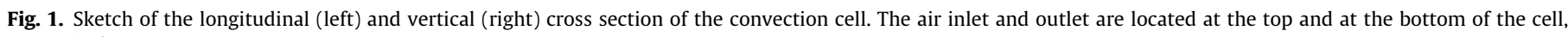
respectively. 
a

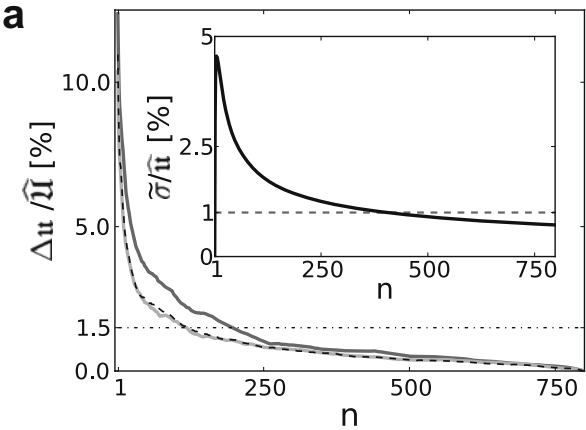

b

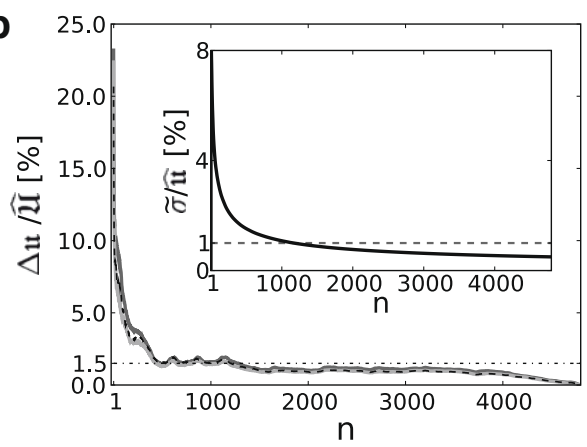

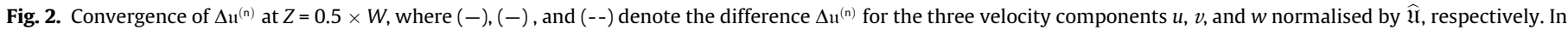
the subplot (-) denotes $\widetilde{\sigma}_{\mathrm{u}}^{(\mathrm{n})}$ normalise by $\widehat{\mathrm{u}}^{(\mathrm{n})}$. (a) FC case at $A r=0$ and $\operatorname{Re}=1 \times 10^{4}$. (b) MC case at $A r=3.3, R a=2.40 \times 10^{8}$ and $\operatorname{Re}=1.01 \times 10^{4}$.

Fig. 2a and b revealing that the convergence criterion $\tilde{\sigma}^{(\mathrm{n})} / \widehat{\mathfrak{u}}^{(\mathrm{n})}<1 \%$ has been fulfilled. For the FC case the criterion is complied for $n \approx 1 /$ $2 \times N$ and for the MC case already for $n \approx 1 / 4 \times N$.

Further, in order to determine the enthalpy balance of the convection cell local temperature measurements have been performed in the smaller cell at 10 bar using platinum resistance thermometers at one position in the air inlet and at five positions in the air outlet, i.e. $X=0.5 \times L, X=0.625 \times L, X=0.75 \times L$, and $X=0.9 \times L$. In the outlet the sensors have been placed $12 \mathrm{~mm}$ downstream behind the exit of the cell. To determine the global temperature difference $\Delta T$ between the cooling and heating plates both have been equipped with platinum resistance thermometers as well. For the measurements at ambient pressure $P \approx 1$ bar and high pressure of $P=10$ bar 25 sensors and 9 sensors have been used on each plate, respectively (Westhoff et al., 2007).

\subsection{Proper Orthogonal Decomposition}

The measured instantaneous velocity fields have been analysed using the Proper Orthogonal Decomposition (POD) technique based on the snapshot method by Sirovich $(1987 a, b)$ to identify coherent structures and their dynamics. For a given set of $N$ two dimensional three component instantaneous velocity fields at a fixed sampling rate

$\underline{U}^{(i)}(\underline{x})=\left(u_{1}^{(i)}, \ldots, u_{l}^{(i)}, v_{1}^{(i)}, \ldots, v_{l}^{(i)}, w_{1}^{(i)}, \ldots, w_{l}^{(i)}\right)$

where $1 \leqslant i \leqslant N$ denotes the time index, $u_{i}, v_{i}$ and $w_{i}$ the velocity components of size $l=m \times n$ ( $m$ is the dimension in $x$-direction and $n$ is one in $y$-direction) the correlation matrix $\underline{\underline{K}}$ of these velocity fields reads

$\underline{\underline{K}}=\frac{1}{N} \underline{\underline{U}} \cdot \underline{\underline{U}}^{T}$

Diagonalising the matrix $\underline{\underline{K}}$ using Singular Value Decomposition (SVD) yields real positive eigenvalues $\lambda_{k}$, ordered in a decreasing order and eigenvectors $\underline{\zeta}_{m}(t)$, which define an orthonormal basis. The linear combination $\underline{\Phi}^{(k)}(\underline{x})$ of the eigenvectors $\zeta_{m}(t)$ and the velocity vectors $\underline{U}^{(i)}$ provides an orthonormal basis with the normalised spatial modes, which can be calculated by

$\underline{\underline{\Phi}}=\underline{\underline{U}} \cdot \underline{\underline{\zeta}}$

$\underline{\Phi}_{i}(\underline{x})$ can be interpreted as spatial kinetic energy distribution, usually termed as coherent structure, and the eigenvectors $\zeta_{i}(t)$ as time series of the dynamics of the coherent structures $\underline{\Phi}_{i}(\underline{x})$ (Sirovich, 1989).

\subsection{Identification of the large-scale circulation axis}

To localise the core as well as the centre of the LSCs a method proposed by Michard et al. (2001) is used. It defines a dimensionless scalar field $\Theta$ for each point $Q$ in a vector field by

$\Theta(Q)=\frac{1}{N} \sum_{S} \sin \alpha_{\mathrm{M}}$,

where $S$ is a two dimensional area surrounding $Q$, and $\alpha_{M}$ denotes the angle between $\underline{u}_{M}$ and the connecting vector of $Q$ and $M$, with $M$ also located in $S$. $\Theta$ is element of the interval -1 and +1 and its sign denotes the direction of the rotation. The scalar $\Theta$ reaches its maximum in the core of the LSC. The advantage of this algorithm is that it allows to determine the location of the centre, the core region and the boundary of the LSCs by considering only the topology of the velocity fields and not its magnitude. Moreover, depending on the size of $S$, turbulent intermittency is filtered and the algorithm is sufficiently robust to process data sets with a large number of instantaneous velocity fields.

\section{Experimental results}

This section is divided into three parts: in the first subsection the results of the temperature measurements under high pressure condition are presented, in the second subsection the formation of the LSC is discussed and in the last subsection the nature of the low-frequency oscillation is analysed by comparing results of temperature measurements under high pressure conditions of 10 bar in the downscaled cell with results of the POD analysis of the vector fields measured in the large cell at ambient pressure.

For calculating the characteristic numbers the height of the cell $H$ was used as the characteristic length and the mean inflow velocity $\widehat{V}_{\text {in }}$ as characteristic velocity $U$. Two flow cases were investigated: FC at $R e=1.0 \times 10^{4}$ and MC at $A r=3.3, R a=2.40 \times 10^{8}$, and $R e=1.01 \times 10^{4}$. PIV has been performed at ambient pressure. All velocities are normalised with the buoyancy velocity $V_{b}=\sqrt{\beta H \Delta T g}=0.58 \mathrm{~m} / \mathrm{s}$ of the MC flow case. For the sake of visibility only every fifth up to every tenth velocity vector is plotted in Figs. 5a, 7a, 9a and 10a.

\subsection{Temperature measurements}

Results of temperature measurements in $\mathrm{MC}$ for $A r=3.3$, $R a=2.40 \times 10^{8}$, and $R e=1.01 \times 10^{4}$ in the smaller cell under high pressure conditions at 10 bar revealed low-frequency oscillations in the heat transfer between the cell inlet and outlet. The frequency and amplitude of this oscillations strongly depends on the magnitude of $R a$ and $R e$, as well as on $A r$. It has been found that in the given rectangular geometry these oscillations occur in a wide range 
of $R a$ and $R e$ for flow cases of $A r>1$. In Fig. 3a part of the temperature fluctuations time series $T_{\text {out }}(t)-\widehat{T}_{\text {out }}$, where $\widehat{T}_{\text {out }}$ denotes the time averaged temperature at $X=0.5 \times L$, is plotted. The plot clearly reveals a periodic oscillation, while the impact on the fluctuations due to variations of the inflow temperature $T_{\text {in }}=27.20(5){ }^{\circ} \mathrm{C}$ is negligible. This oscillation of the temperature at the outlet has also been found at $X=0.625 \times L$ and $X=0.75 \times L$, but not at the outlet position $X=0.90 \times L$. The reason for this might be the influence of the lateral boundary. The corresponding power spectrum of the temperature fluctuations at $X=0.5 \times L$ and $X=0.75 \times L$ is shown in Fig. $4 \mathrm{a}$ and b. The $x$-axis in these plots is scaled logarithmically. Two characteristic frequencies are observed in the power spectra: a very low characteristic frequency $\omega_{\text {low }}=0.008 \mathrm{~s}^{-1}$ and a higher one at $\omega=0.32 \mathrm{~s}^{-1}$. These oscillations are strongly tied to the formation and dynamics of the LSCs, as will be shown in the following.

\subsection{Large-scale structure formation}

The measured velocity fields for FC in the centre cross section $X=0.5 \times L$ are presented in Fig. 5a. Clearly, the jet of incoming air from the inlet (upper right corner) can be identified. This inflow drives a nearly two-dimensional and stationary rotating mean wind, whose core is located near the centre of the cross section. The velocity profiles of the $v$-component at $Y=0.55 \times H$ in the cross sections ( Fig. 5b) reveal the two-dimensional nature of the mean wind. Fig. $6 \mathrm{~b}$ depicts the standard deviation $\sigma_{w}$ of the $w$ component, which reveals a rather homogeneous distribution indicating the rather stationary nature of the flow in the core region of the roll structure, with $\sigma_{w}$-values less than $0.06 \times V_{\mathrm{b}}$. Only at the top elevated $\sigma_{w}$-values can be found. There, the wall jet detaches and starts oscillating as a result induced by the instability of the free shear layer which develops between air jet and the LSC. From the linear dependence of the radial velocity component $v$ on the radius depicted in Fig. $5 \mathrm{~b}$ it can be concluded that the mean wind behaves like a solid body (Rankine vortex) in nearly the whole cross section with a angular frequency of $\omega_{F C}=0.66 \mathrm{~s}^{-1}$. Additionally in the core region of the LSC a wave structure with a wavelength of $\lambda=L \cdot\left(\Gamma_{x z}\right)^{-1}$ in longitudinal direction ( $L$ denotes the length of the cell), is observed. This wave structure is also visible in the isolines Fig. 6a, where the velocity component $w$ is gray-scaled. The in-plane velocity components are not plotted in this figure because they are very small in comparison to the out-of-plane velocity component.

Further, it has to be noted that in other measurements of FC for $1.0 \times 10^{4}<\operatorname{Re}<2.56 \times 10^{4}$ wave structures with the same wave length have been observed. The amplitude of this wave structure depends on the inflow velocity and increasing inflow velocities lead to higher amplitudes. We ascribe this structure to the given aspect ratio $\Gamma_{x z}=5$ of the cell, which leads to a wavelength of $\lambda=L / 5$.

For MC at $A r=3.3$ the flow is strongly dominated by buoyancy forces. Due to the density gradient between the heating and cooling plates LSCs oriented in longitudinal direction develop. This is in contrast to the velocity field in the longitudinal cross section at $Z=0.5 \times X$ for pure FC (Fig. 6a) where the in-plane velocity components are negligible compared to the out of plane component. But considering the entire measurement plane, the in-plane velocity components are now of the same order as the out-of-plane velocity component. Fig. 7a reveals one of four longitudinal arranged LSCs. These LSCs have been also found in direct numerical simulations of pure $\mathrm{RBC}$ in a rectangular container with the same aspect ratio, but

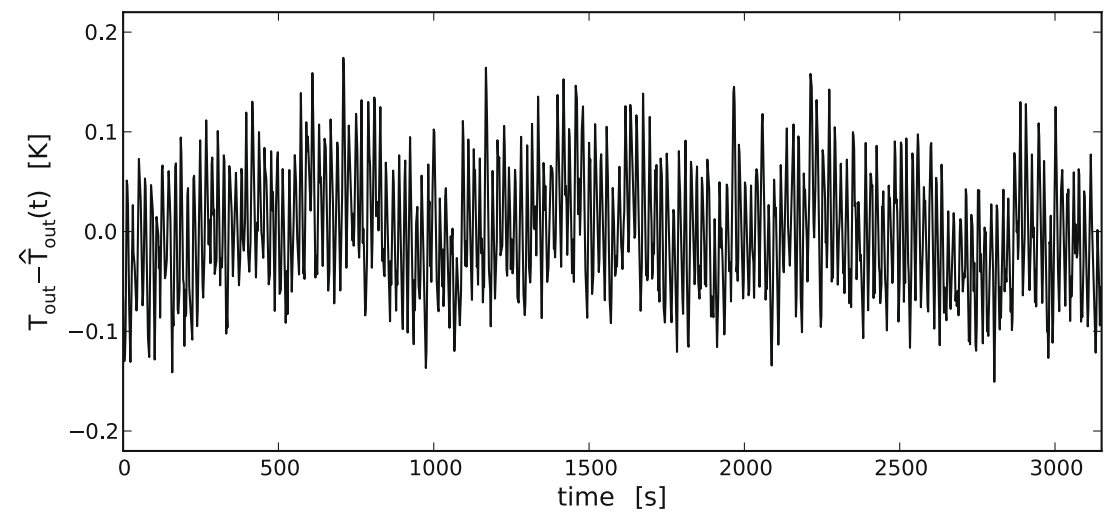

Fig. 3. Fluctuations of the temperature time series at the outlet of the cell at $X=0.5 \times L$ measured in the small cell at 10 bar for the MC case.

a

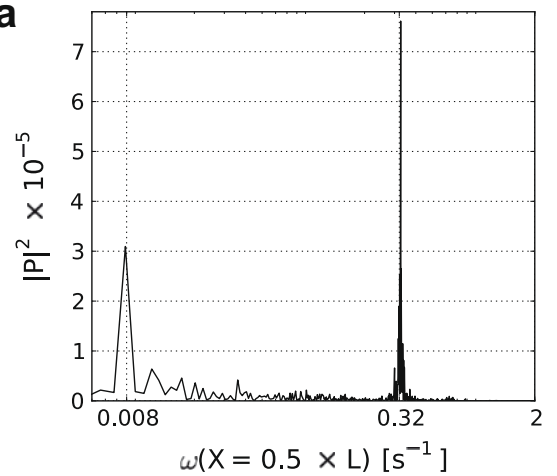

b

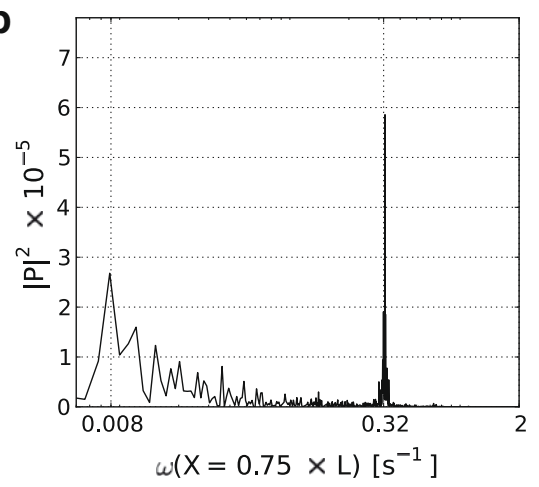

Fig. 4. Power spectra of the temperature time series at the outlet of the cell at $X=0.5 \times L$ (a) and $X=0.75 \times L$ (b). 
without air in- and outlet by Kaczorowski and Wagner (2008). In contrast to the LSCs found in the simulations in purely TC, where the LSCs are arranged in longitudinal cell direction, an angular shift of the LSCs is observed in MC due to the superposition with FC. This can be concluded from the velocity fields depicted in Figs. 9 and 10, which reveal a remaining, yet asymmetrical, roll structure in the cross cut planes $X=0.5 \times L$ and $X=0.75 \times L$. In Fig. 8b, where the $v$-component of the longitudinally oriented velocity field is plotted, a region of rising hot air $(X \approx 0.78)$ and descending cold air
$(X \approx 0.54)$ is found. The corresponding values of the $u$-component are presented in Fig. 8a, revealing the high level of symmetry of the LSCs in MC. The breakdown of the two-dimensional stationary roll structure found in FC into a more complex, instationary pattern of LSCs in MC is reflected by the increase and structural change of the $\sigma$-distribution, depicted in Fig. 7b. Here $\sigma$ denote the standard deviation of the velocity magnitude. Particularly in the region very close to the top, where the horizontal flow of the buoyancy induced LSCs (Fig. 8a) interacts with the wall jet, the highest fluctu-
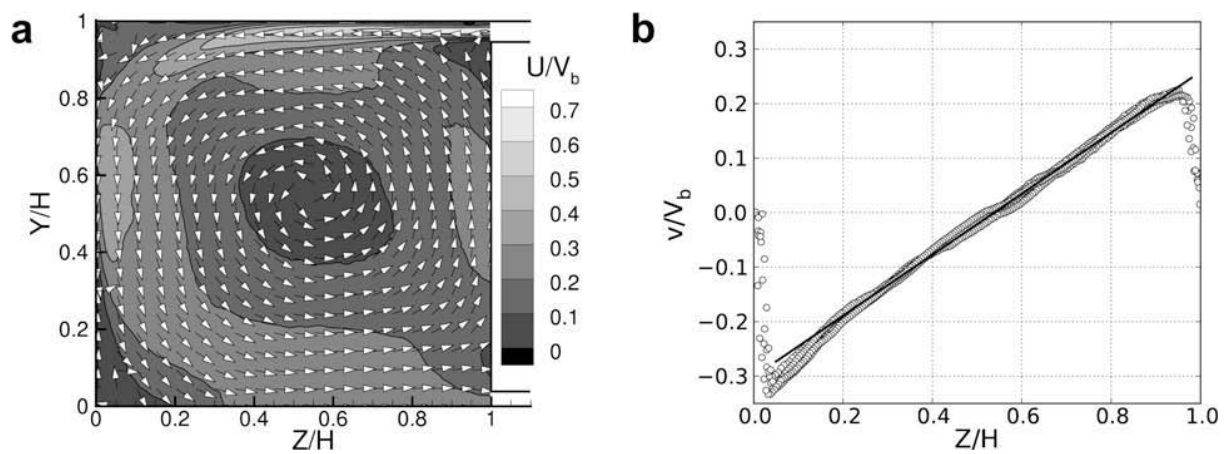

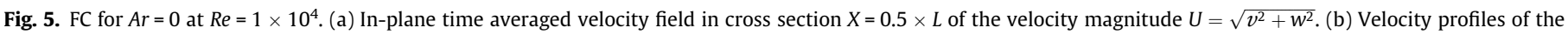
$v$-component at $Y=0.55 \times H$ in the vertical cross sections $X=0.5 \times L, X=0.625 \times L, X=0.75 \times L$ and $X=0.9 \times L$.
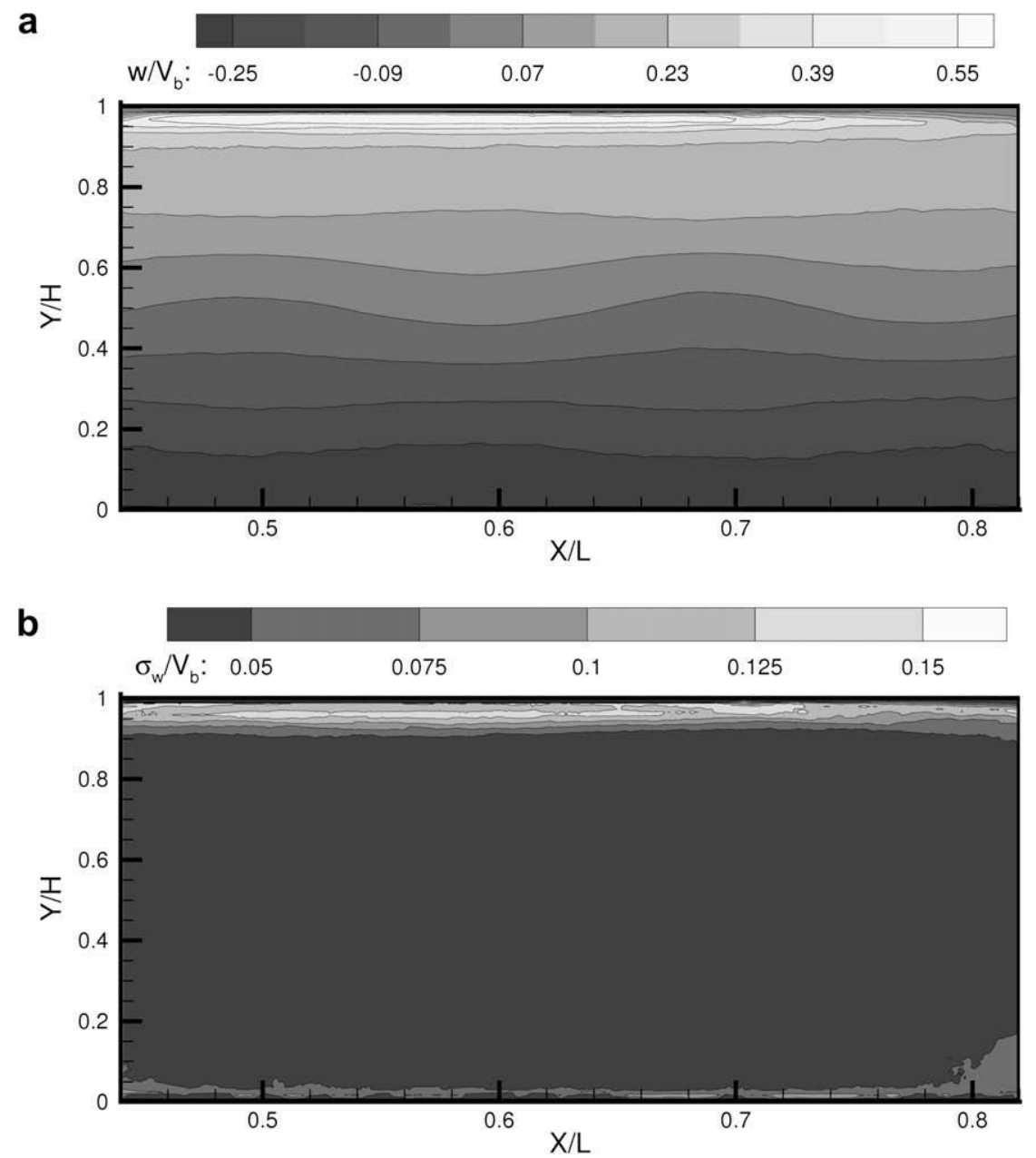

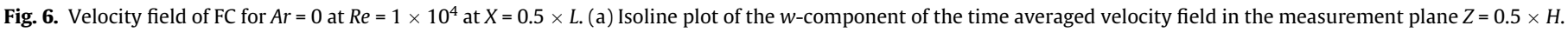
(b) Isoline plot of the standard deviations of the velocity component $w$.

Please cite this article in press as: Westhoff, A., et al. Experimental study of low-frequency oscillations and large-scale circulations in turbulent mixed convection. Int. J. Heat Fluid Flow (2010), doi:10.1016/j.ijheatfluidflow.2010.04.013 
ations emerge. Locally increased velocity fluctuations can be found as well in the region of the rising hot air at $X \approx 0.78 \times L$, while in the region of descending cold air very low $\sigma$-values are observed. We ascribe this difference to the fact that the plumes, which drive the LSCs, are convected by the wall jet at the cooling plate. This leads to a change in dynamics and a different temperature stratification close to cooling plate, where the jet is faster in the vicinity of the heating plate and the wall jet has already grown in thickness.

Simultaneously a centre shift of the LSC in the vertical measurement plane takes place. The shift of the centre of this LSC depends strongly on the position of the cross section in $x$-direction. In the region of descending air the centre is shifted towards the upper right corner (Fig. 9a), while in the region of rising plumes it is shifted towards the upper left corner (Fig. 10a). The reason of the centre shift at $X=0.5 \times L$ is the primarily downward oriented flow in this region (Fig. 7a). The falling cold plumes are convected away by the incoming wall jet and the additional momentum of these plumes leads to increased velocities of the downward oriented flow. As a consequence the downward flow in the region of the left side wall is broadened (Fig. 9c), while the upward flow is constrained to a very narrow region at the right side wall. In the cross section at $X=0.75 \times L$ the vertical flow is dominated by rising fluid (Fig. 9c). Hence, opposite to the flow in the cross section at $X=0.5 \times L$ the region at the right side wall, where the up-flow dominates, is strongly enlarged and the downward flow is localised close to left side wall. In addition, compared to the flow in cross section $X=0.5 \times L$, in the cross section $X=0.75 \times L$ the rising plumes trigger a deferred detachment of the wall jet at the top, which in turn leads to a significant increase of the velocity fluctuations in the whole cross section (Fig. $9 \mathrm{~b}$ and 10b). In the cross sections $X=0.75 \times L$ and $X=0.5 \times L$ locally elevated values of $\sigma$ are found in the regions where the wall jet detaches and close to the heated bottom plate. Additionally, in the cross section $X=0.5 \times L$ alternating $\sigma$-values at the region above the heated plate are observed (Fig. 9b). We ascribe this to the occurrence of preferred points of plume emission at the bottom plate. The momentum of this plumes is not large enough to rise in this cross section. Due to the interaction with the flow over the heating plate, which is heading to the outlet, these structures are damped and washed away in direction to the outlet, as well towards of $X \approx 0.78 \times L$ where hot air rises.

For further analysis of the LSCs and their influence on the heat transfer the topology of the velocity fields is analysed using the LSC identification algorithm described in Section 2.4. The statistical distribution of the LSC centre position in the cross sections $X=0.5 \times L$ and $X=0.75 \times L$ shown in Fig. 11. Fig. $11 \mathrm{~b}$ and $d$ reveals the scalar field of $\Theta(P)=\frac{1}{N} \sum_{S} \sin \alpha_{\mathrm{M}}$ calculated from the time averaged velocity field. Positive values represent counter clockwise and negative values clockwise rotation. The absolute values are a measure for the proximity to the LSC core centre and the maximum absolute values represent the centre of a LSC. As already discussed above Fig. 11b and d reflect as well the shift of the LSC towards the
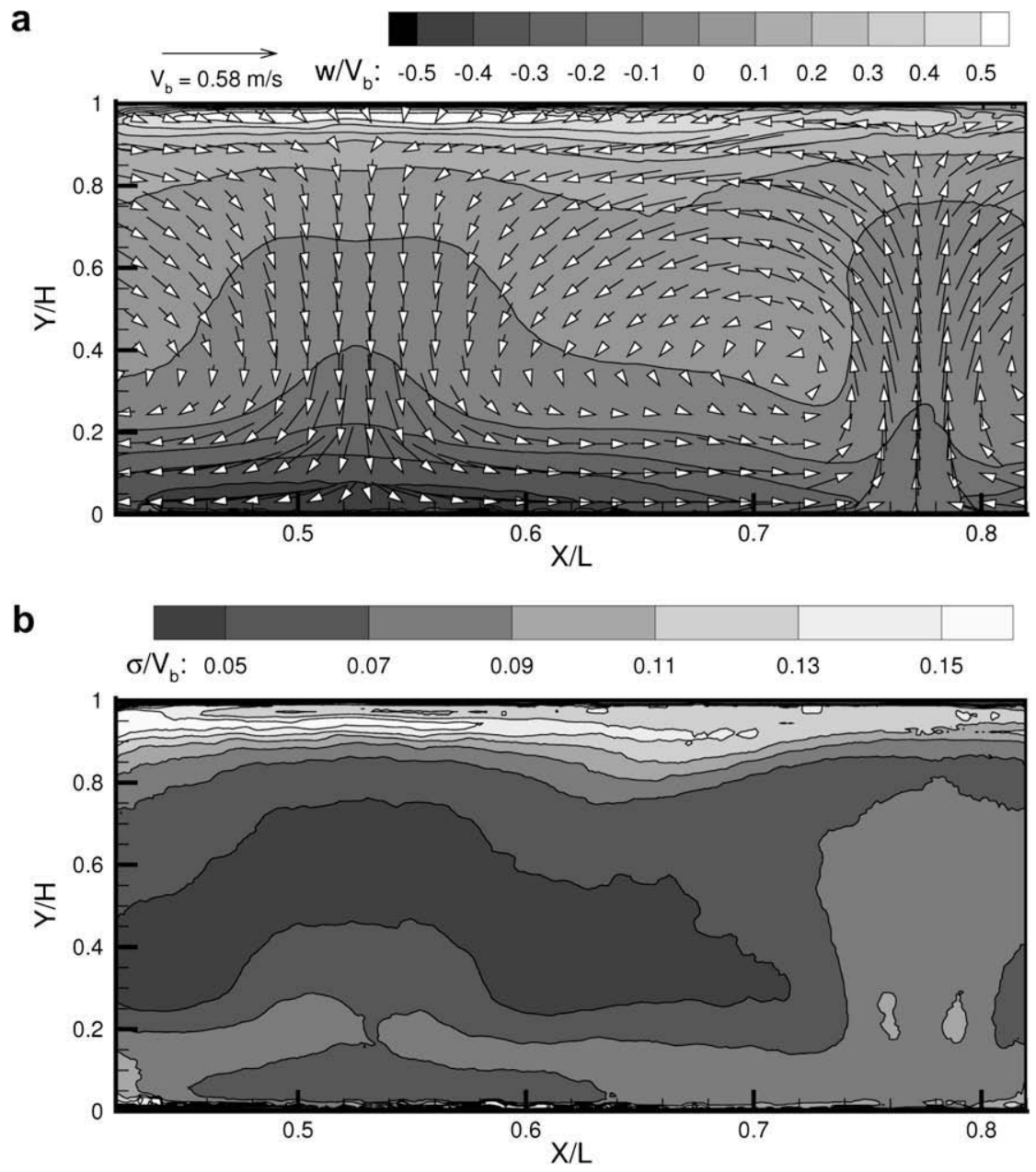

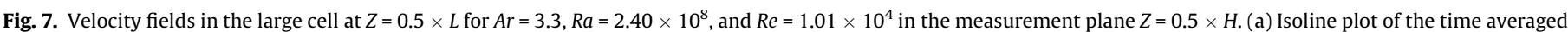

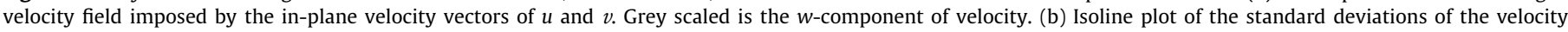
magnitude. 
upper right corner for the region of falling plumes and the upper left corner for the region of rising plumes, respectively. The plots further show that the LSCs are not completely rotationally symmetrical any more. Moreover, an eccentricity in $y$-direction for the LSCs in cross section $X=0.5 \times L$ and in $x$-direction to the upper left corner in cross section $X=0.75 \times L$ can be observed. This is the result of the rising and falling plumes, which strongly influence the dynamics of the forced convective induced LSCs for $A r=3.3$. The eccentricity in the cross section $X=0.5 \times L$, with descending cold plumes, is not as pronounced as in the cross section $X=0.75 \times L$, where hot rising plumes can be observed.

Additionally to the topology of the LSC the statistical distribution of the centre positions are presented in Fig. 11a and c. The black circles reflect the position of the LSC centres for each instantaneous vector field and the histograms depict the corresponding frequency distribution in $x$ - and $z$-direction with the number $N$ the counting how often the core centre has located on this position. In the cross section $X=0.5 \times L$ (Fig. 11a) the centre is located in the interval of $Z / H \approx[0.20,0.90]$ and $Y / H \approx[0.2,0.80]$, however a cumulation is detected in the region of $Z / H \approx[0.70,0.82]$ and $Y \mid$ $H \approx[0.55,0.75]$. The movement of the LSC centre in the cross section at $X=0.75 \times L$ is shown in Fig. 11c. It is located at $Z /$ $H \approx[0.15,0.70]$ and $Y / H \approx[0.3,0.85]$, and a cumulation is detected in the region of $Z / H \approx[0.20,0.4]$ and $Y / H \approx[0.50,0.8]$. Considering the frequency distribution of the centre position in the $x-z$ plane in Fig. 11c it is observed that the LSCs are more localised in the region of falling plumes as compared to the cross section where rising plumes dominate. In both cross sections the frequency distributions are asymmetrical in $y$ - and $z$-direction. This is the result of the non-central position of the mean LSC centre and of the influence of the wall leading to more asymmetric LSCs.

\subsection{Low-frequency oscillations}

Finally the power spectrum of the eigenvectors $\zeta_{k}$ obtained from the POD analysis of the velocity vector fields measured at ambient pressure in the large cell in the cross section $Z=0.5 \times H$ is compared to the power spectrum of a temperature time series measured in the downscaled container under high pressure condition. The POD analysis has been performed by using 4800 velocity fields for the MC case and 800 for the FC case with a spatial resolution of $4.5 \mathrm{~mm}$ in $x$ - and $y$-direction. Both measurements, that in the large cell at ambient pressure and that in the smaller cell under high pressure conditions, have been performed for the same characteristic numbers $A r=3.3, R a=2.40 \times 10^{8}, R e=1.01 \times$ $10^{4}$ and $\operatorname{Pr}=0.7$.

Analysing the measured temperature data spatial temperature variations over the length of the outlet depending on the orientation of the buoyancy induced LSCs have been found. In regions of rising plumes the time averaged outlet temperature $\widehat{T}_{\text {out }}$ is higher and in regions with predominantly falling plumes $\widehat{T}_{\text {out }}$ is lower. Such oscillations have been observed over a wide range of $R e$ and $R a$ for $A r>1$. The corresponding power spectra of the temperature fluctuations at the outlet are presented in Fig. 4. The figures reveal the two characteristic frequencies, located at $\omega=0.32 \mathrm{~s}^{-1}$ and $\omega_{\text {low }}=0.008 \mathrm{~s}^{-1}$. But considering these frequencies with the angu-
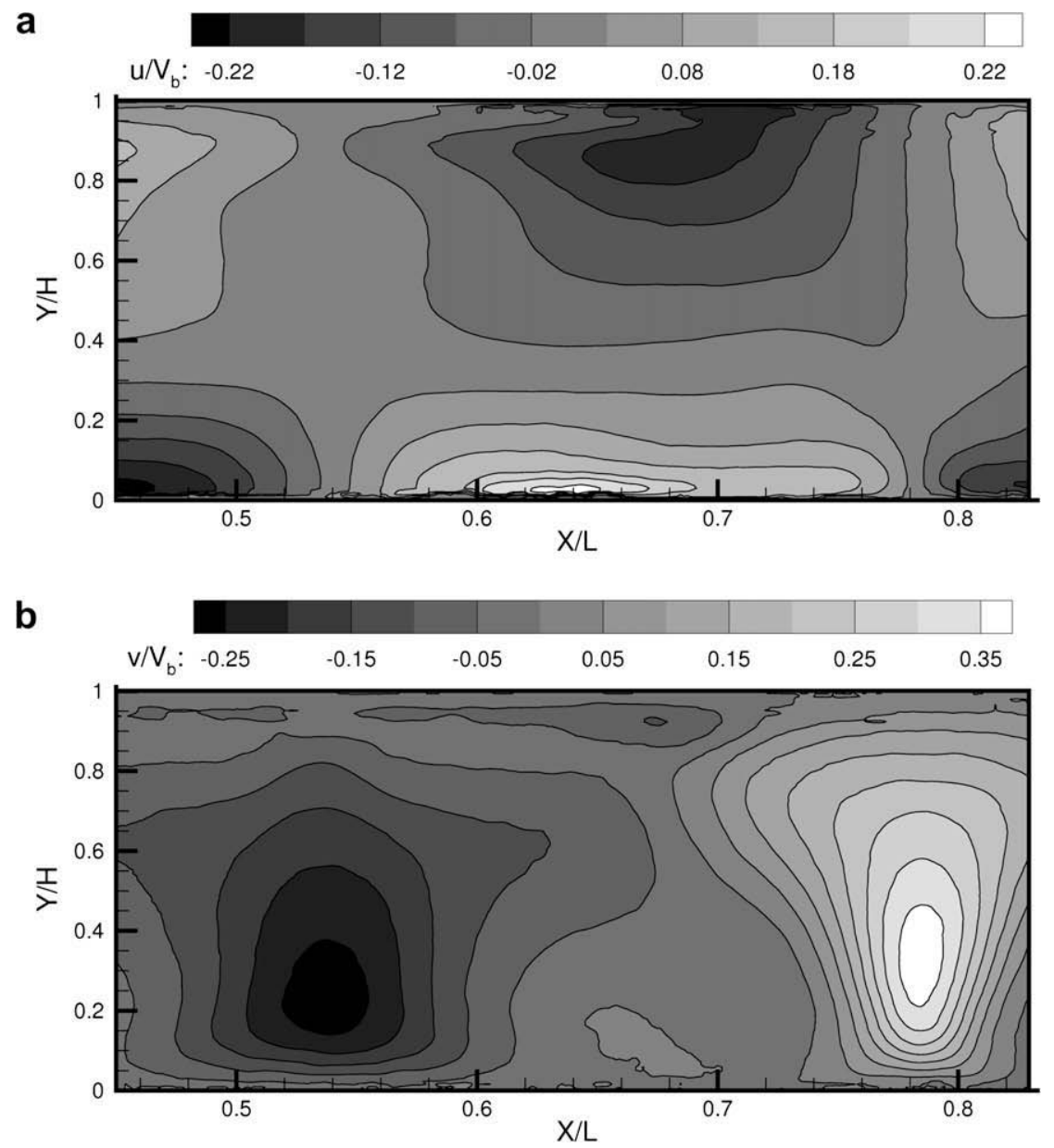

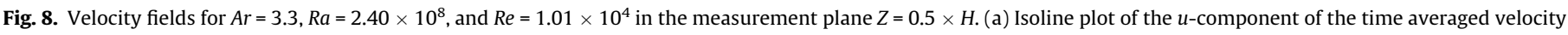
field. (b) Isoline plot of the $v$-component of the time averaged velocity field. 

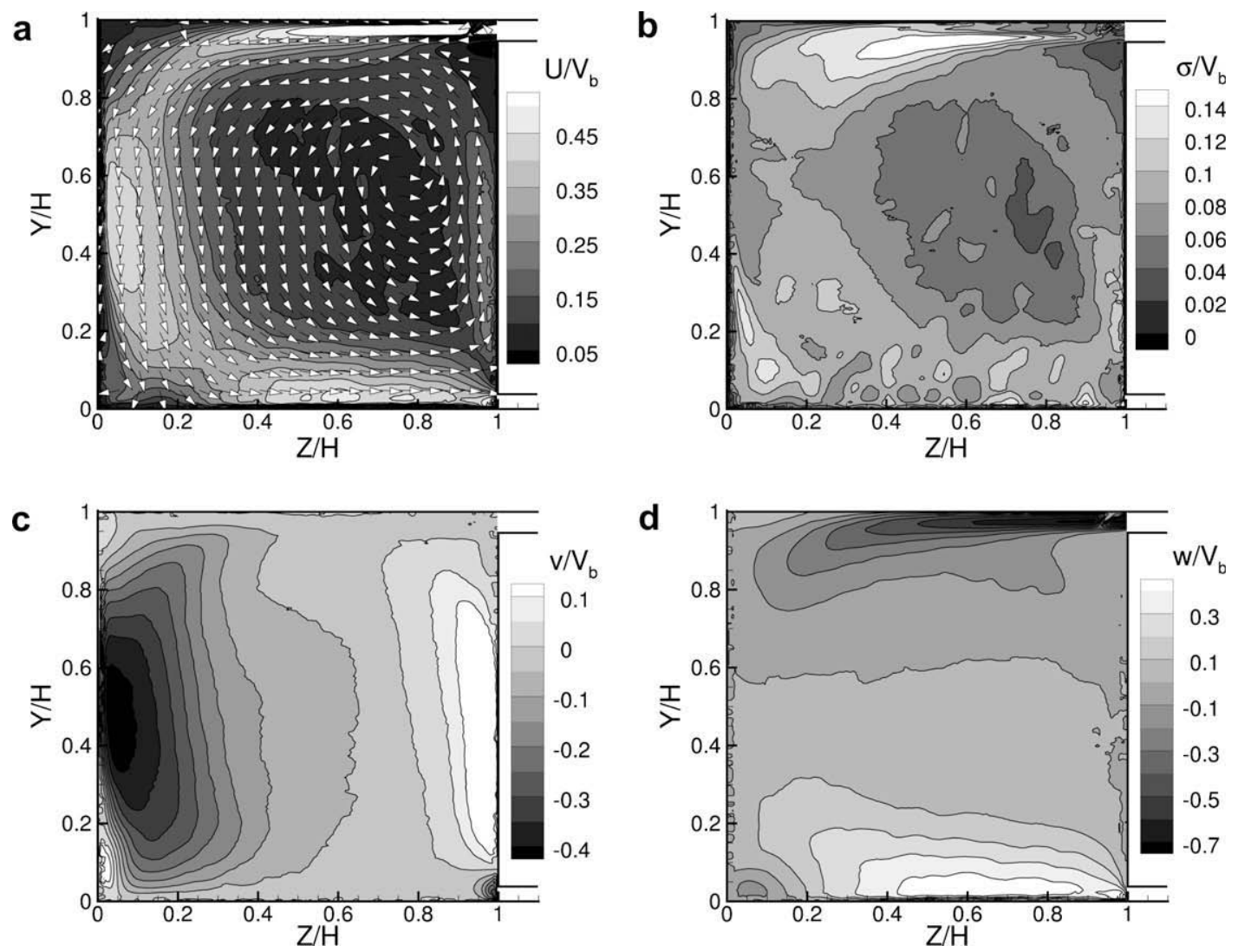

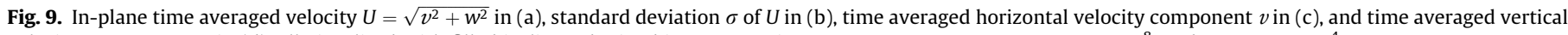
velocity component $w$ in (d), all visualised with filled isolines obtained in cross section $X=0.5 \times L$ at $A r=3.3, R a=2.40 \times 10^{8}$, and $R e=1.01 \times 10^{4}$.
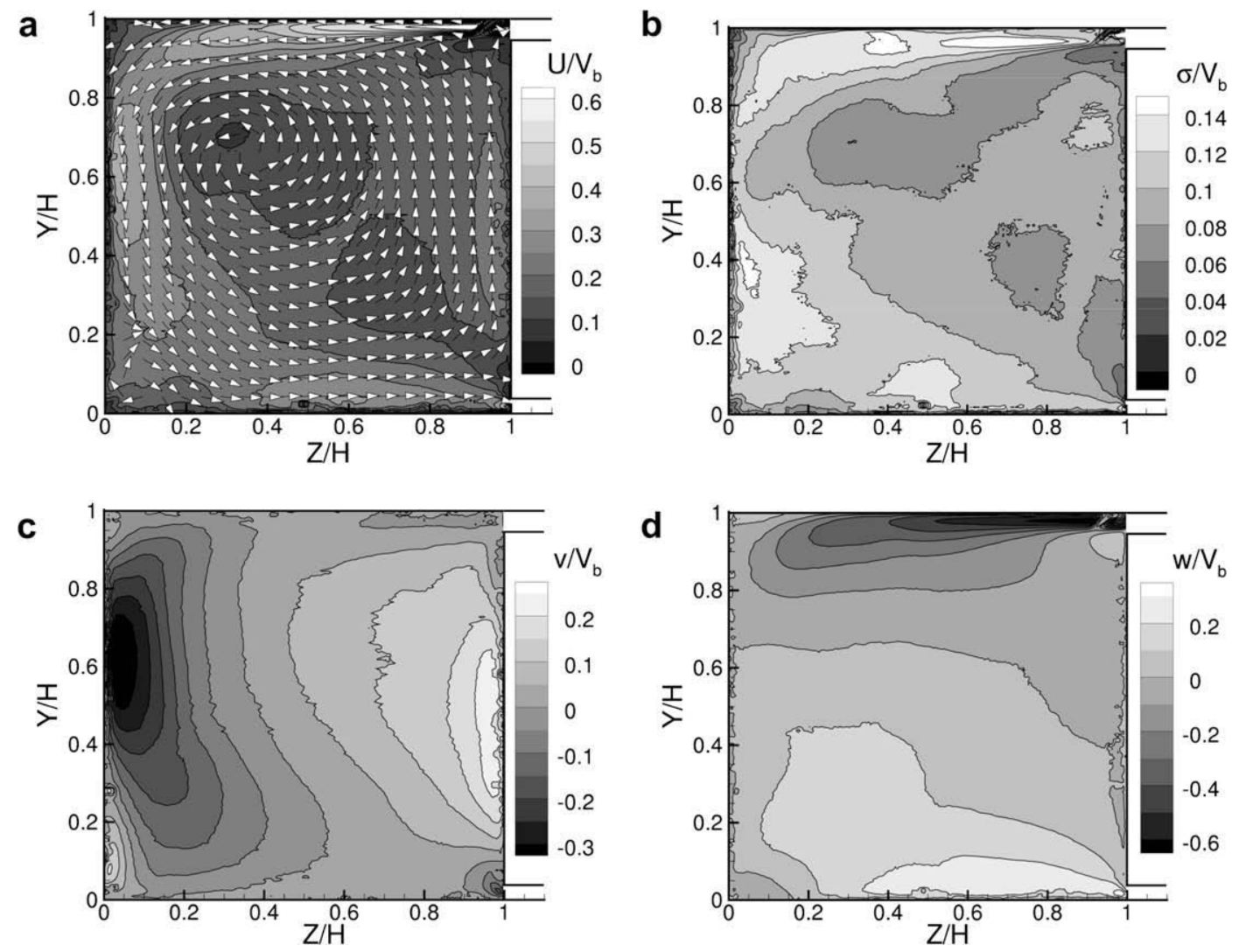

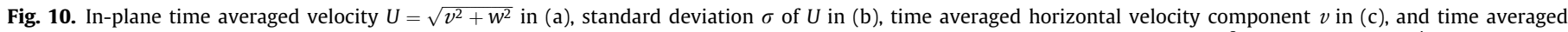
vertical velocity component $w$ in $(\mathrm{d})$, all visualised with filled isolines obtained in cross section $X=0.75 \times L$ at $A r=3.3, R a=2.40 \times 10^{8}$, and $R e=1.01 \times 10^{4}$. 
lar frequency of the LSCs measured in MC it follows that the higher frequency $\omega$ equals the average angular frequency $\omega_{\mathrm{TC}} \approx 0.32 \mathrm{~s}^{-1}$ of the longitudinal roll structure found in Fig. 7, which has been evaluated from horizontal and vertical line scans of the LSC. The second characteristic frequency $\omega_{\text {low }}$ (Fig. 4) is much lower than the angular frequency associated to the vertical LSC of the FC case $\omega_{\mathrm{FC}} \approx 0.66 \mathrm{~s}^{-1}$ presented in Fig. 5 and the angular frequency $\omega$ of the longitudinal arrange LSC. To investigate if the above frequencies are related to the dynamics of any coherent flow structures a POD analysis of the PIV data obtained for forced and mixed convection has been performed. For the longitudinal measurement plane $Z=0.5 \times H$ in the MC case the first POD mode contains $73 \%$ of the total energy and represents the LSC. The eigenfunction is not shown here because its topology corresponds to that of the time averaged velocity field depicted in Fig. 7a.

The convergence of the normalised eigenvalue sum $\tilde{\lambda}$ and the decay of the eigenvalues $\lambda_{n}$ as function of the mode number $n$ are presented in Fig. 13. The high value of the first eigenvalue $\lambda_{1}$ shows that for MC a coherent structure exists which contains most of the kinetic energy of the flow. All other eigenvalues $\lambda_{i}$ are much lower and contain less than $1 \%$ of the total energy. They represent superimposing periodically and non-periodically fluctuating coherent structures. We conclude from this that the flow is dominated by the coherent structure related to the first mode.

Comparing the power spectra of the temperature time series obtained in the high pressure case (Fig. 4) with the power spec-

\section{a}

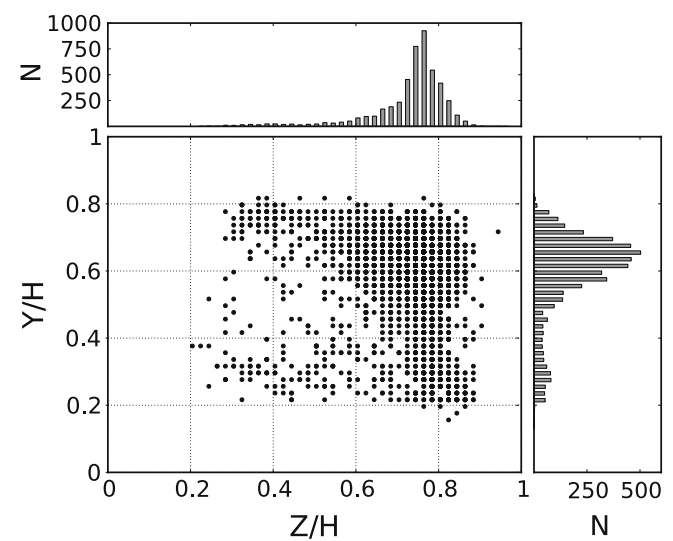

C

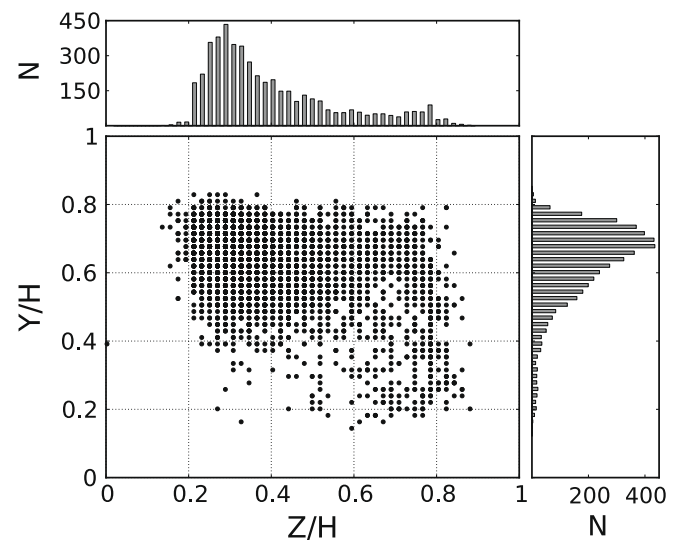

b

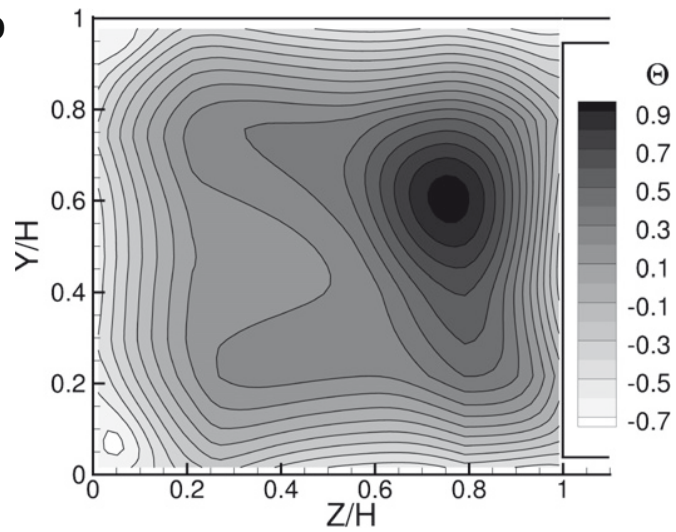

d

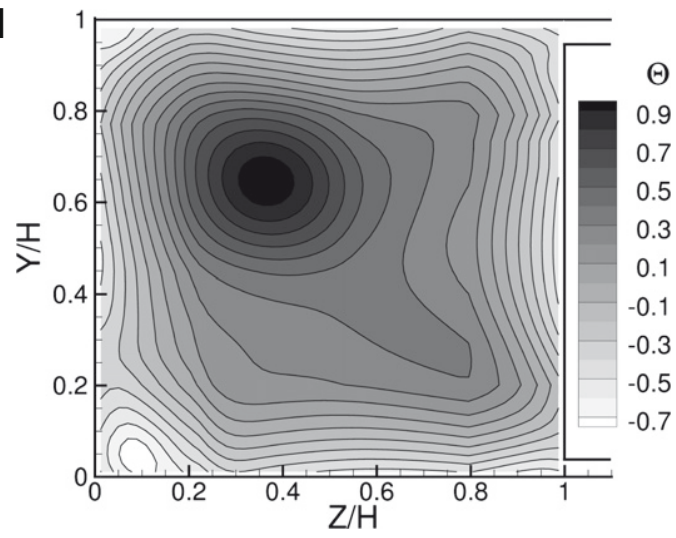

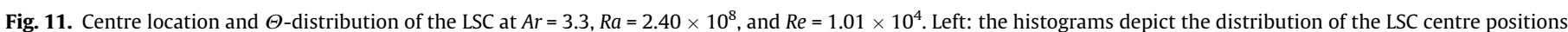

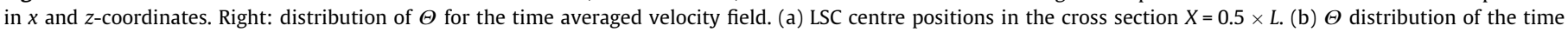
averaged velocity field at $X=0.5 \times L$. (c) LSC centre positions in the cross section at $X=0.75 \times L$. (d) $\Theta$ distribution of the time averaged velocity field at $X=0.75 \times L$.
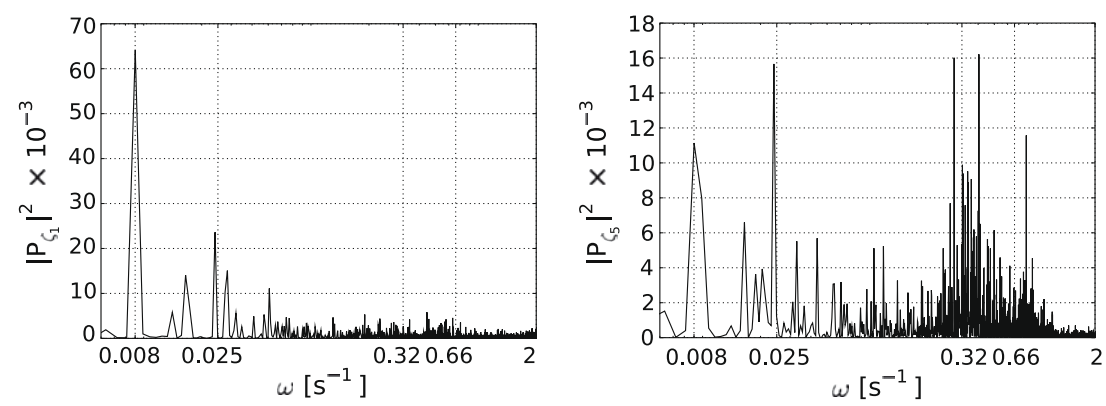

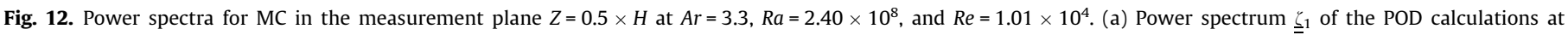
$Z=0.5 \times H$. (b) Power spectrum $\zeta_{5}$ of the POD calculations at $Z=0.5 \times H$. 


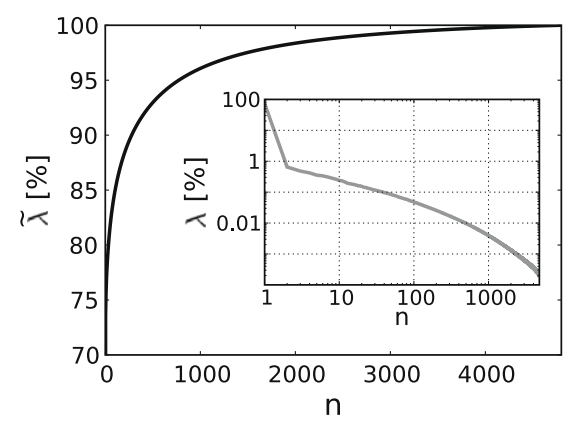

Fig. 13. Convergence of the normalised eigenvalue sum $\tilde{\lambda}$ as function of the mode number $n$ in cross section $Z=0.5 \times H$ for the MC case $\left(A r=3.3, R a=2.40 \times 10^{8}\right.$, and $\left.R e=1.01 \times 10^{4}\right)$. The inset depicts the eigenvalues as function of the mode number; both axes are scaled logarithmically.

trum of the eigenvectors of the two most relevant modes plotted in Fig. 12 the same characteristic frequencies are found. The power spectrum $\zeta_{1}$ of FC (not shown here) reveals no characteristic frequency, which compares to the characteristic frequencies found in the power spectrum of the outlet temperature time series in $\mathrm{MC}$ at the outlet position $X=0.5 \times L$ and $X=0.75 \times L$. This is in contrast to the power spectrum of the eigenvectors $\underline{\zeta}$ for MC. The rather low frequency of $\omega_{\text {low }}=0.008 \mathrm{~s}^{-1}$ can be observed in many power spectra $\zeta$ of the first 10 POD modes. Why this low-frequency oscillation develops is not yet fully understood. However, we assume that the observed oscillations are a result of rotational or even torsional oscillations of the LSCs, which have been observed before in pure RBC, e.g. by Funfschilling et al. (2008). The other characteristic frequencies at $\omega \approx 0.32 \mathrm{~s}^{-1}$, found in the power spectrum of the temperature in several power spectra of $\zeta$ of the first 10 POD modes, e.g. for $\zeta_{5}$ presented in Fig. 12b. However, in the power spectrum of the eigenfunctions $\zeta_{5}$ not a single but rather a variety of sharp peaks located around $\omega$ peak exist. Finally we want to emphasize that the same characteristic frequencies have been observed in two independent measurements.

\section{Conclusions}

An experimental study of the formation and dynamics of LSCs in FC for $R e=1.01 \times 10^{4}$ and $\operatorname{Pr}=0.7$ and $\mathrm{MC}$ for $A r=3.3$, $R a=2.40 \times 10^{8}, R e=1.01 \times 10^{4}$ and $P r=0.7$ has been presented. The experiments were conducted in two rectangular cavities with the same geometry: the larger one was operated at ambient fluid pressure and the smaller one (downscaled by a factor 5 ) under high pressure conditions. PIV in the large container and temperature measurements in the smaller one have been performed.

Temperature measurements under high pressure conditions revealed low- and very low-frequency oscillations of the temperature at the cell outlet. These oscillations occur at elevated $\mathrm{Ar}$. Only for the MC case, in which buoyancy forces start to dominate the flow, these oscillations have been observed. It was further shown that the frequency and amplitude of the oscillations depend on the magnitudes of $R a$ and $R e$. The dependence of these frequencies on $\mathrm{Ar}$ has been investigated in the past and are discussed in Westhoff et al. (2008).

Knowing, that the transport of heat in our configuration is strongly correlated with the formation and dynamics of LSCS PIV has been performed in $\mathrm{MC}$ in order to identify fingerprints of these low-frequency oscillation in the velocity fields. To make sure that the low-frequency oscillations found at the temperature measurements at 10 bar are not induced by the wind tunnel or the experimental set-up itself the PIV measurement were conducted in the large cell which provides a much better optical accessibility as compared to the smaller one mounted in the high pressure wind tunnel.

Regarding FC it has been found that for $R e=1.01 \times 10^{4}$ a quasi two-dimensional roll structure develops. The core of this LSC induced by the pressure gradient between the inlet and the outlet behaves like a solid body rotation and is nearly stationary in time. In MC a breakdown of this two-dimensional roll structure has been observed as a result of the cross flow caused by the temperature gradient between the bottom and the ceiling at $A r=3.3$. Moreover, the formation of four longitudinally arranged quasi two-dimensional roll structures has been found. To determine the interaction of these LSCs the velocity fields have been analysed using a LSC detection algorithm. It was shown that depending on the position in longitudinal direction the LSC core and core centre is shifted towards the upper left or right direction. The direction of the shift strongly correlates with the thermal plume motion. In the region of falling cold air the core shifts towards to the right side wall, where the inlet and outlet port is located, while in the region of rising hot air the core is shifted the to the left sidewall. Further it was shown that, as a function of time, the core centre position moves in a wide range of the cross section.

To analyse in more detail how the low-frequency oscillations of the temperature at the outlet are related to the dynamics of the LSCs a POD study of the instantaneous velocity fields in longitudinal cross section has been performed. The POD analysis revealed the existence of a coherent structure which containing $73 \%$ of the total kinetic energy. This coherent structure represents the LSC oriented in longitudinal direction. The power spectrum of the corresponding eigenvector reflects the same low-frequency oscillations as those obtained from the temperature measurement. We assume that this oscillation is the result of rotational or even torsional oscillations of the LSCs, which have been observed before in RBC. The other characteristic frequency found in the temperature measurements equals the angular frequency of the longitudinally arrange LSC. This frequency has been also found in the eigenvector power spectrum of the fourth POD mode. Moreover, we believe that this coherent structure is related to a nearly periodic plume emission from the boundary layers which drives the LSCs in longitudinal direction.

At last, this experimental study of $\mathrm{MC}$ is also an interesting example for the organisation of random unstable modes in a nearly deterministic system in space and time. As a result large-scale structures are formed. Moreover, these structures rotate in a nearly coherent manner.

\section{References}

Costa, J.J., Oliveira, L.A., Blay, D., 2000. Turbulent airflow in a room with a two-jet heating-ventilation system - a numerical parametric study. Int. J. Heat Mass Transfer 32, 327-343.

Funfschilling, D., Ahlers, G., 2004. Plume motion and large-scale circulation in a cylindrical Rayleigh-Bénard cell. Phys. Rev. Lett. 92 (19), 194502.

Funfschilling, D., Brown, E., Ahlers, G., 2008. Torsional oscillations of the large-scale circulation in turbulent Rayleigh-Bénard convection. J. Fluid Mech. 607, 119139.

Kaczorowski, M., Wagner, C., 2008. Analysis of the thermal plumes in turbulent Rayleigh-Benard convection based on well-resolved numerical simulation. J. Fluid Mech. 618, 89-112.

Kupka, F., 2003. Convection in stars. In: Proceedings Int. Astronomical Union Symposium, pp. 119-129.

Linden, P.F., 1999. The fluid mechanics of natural ventilation. Annu. Rev. Fluid Mech. 31, 201-238.

Michard, M., Graftieaux, L., Grosjean, N., 2001. Combining PIV, POD and vortex identification algorithms for the study of unsteady turbulent swirling flows. Meas. Sci. Technol. 23, 1422-1429.

Niemela, J.J., Skrbek, L., Sreenivasan, K.R., Donnelly, R., 2001. The wind in confined turbulent convection. J. Fluid Mech. 449, 169-178.

Qui, X.-L., Tong, P., 2001a. Large-scale velocity structures in turbulent thermal convection. Phys. Rev. E 64, 036304.

Qui, X.-L., Tong, P., 2001b. Onset of coherent oscillations in turbulent RayleighBénard convection. Phys. Rev. Lett. 87 (9), 094501.

Schmeling, D., Westhoff, A., Kühn, M., Bosbach, J., Wagner, C., 2010. Flow structure formation of turbulent mixed convection in a closed rectangular cavity. In: 
Notes on Numerical Fluid Mechanics and Multidisciplinary Design (NNFM), vol. 112.

Sillekens, J., Rindt, C., Steenhoven, A., 1998. Developing mixed convection in a coiled heat exchanger. Int. J. Heat Mass Transfer 41, 61-72.

Sirovich, L., 1987a. Turbulence and the dynamics of coherent structures, part I: coherent structures. Quart. Appl. Math. 45 (3), 561-571.

Sirovich, L., 1987b. Turbulence and the dynamics of coherent structures, part II: symmetries and transformation. Quart. Appl. Math. 45 (3), 573-582.
Sirovich, L., 1989. Chaotic dynamics of coherent structures. Phys. D 37, 126-145.

Westhoff, A., Grabinski, N., Bosbach, J., Wagner, C., Thess, A., 2007. Scaling of turbulent mixed convection under high pressure. In: Fifth International Symposium on Turbulence and Shear Flow Phenomena, vol. 2, pp. 505-510.

Westhoff, A., Bosbach, J., Wagner, C., 2008. Scaling of mixed convection in aircraft cabins. In: 26th International Congress of the Aeronautical Sciences.

Xia, K.Q., Sun, C., Zhou, S.-Q., 2003. Particle image velocimetry measurements of the velocity field in turbulent thermal convection. Phys. Rev. E 68, 066303. 